\title{
Orbital and Ocular Trauma at a Tertiary Care Centre - A Clinical Profile
}

\author{
Mini P.A. ${ }^{1}$, Viswakala V.S. ${ }^{2}$, Josey Verghese T. ${ }^{3}$ \\ ${ }^{1}$ Department of Ophthalmology, Government TD Medical College, Alappuzha, Kerala, India. ${ }^{2}$ Department of \\ Community Medicine, Government TD Medical College, Alappuzha, Kerala, India. ${ }^{3}$ Department of Radio \\ Diagnosis, Government TD Medical College, Alappuzha, Kerala, India.
}

\section{ABSTRACT}

\section{BACKGROUND}

One of the major causes of preventable blindness is ocular trauma, which also results in high socioeconomic burden. This study was done to understand the clinical profile of orbital and ocular trauma at a tertiary care centre in Kerala.

\section{METHODS}

A descriptive study was conducted among patients attending the casualty of a tertiary care centre in Kerala for a period of three months from August 2017 to October 2017. A semi structured proforma was filled by the ophthalmologist attending the patients. This data was analysed using appropriate software. The study included a total of 256 patients with orbital and ocular trauma. After taking a detailed history, visual acuity testing and ocular examination were conducted. Computed tomography (CT) scan of orbit was done whenever indicated.

\section{RESULTS}

Out of 256 patients, $60.9 \%$ were in the age group of 21 - 60 years. Males were affected about 4 times more than the females. $40 \%$ of trauma occurred in the afternoon between 12 and $6 \mathrm{pm}$, and $29 \%$ happened from $6 \mathrm{pm}$ to 12 midnight. Majority of trauma ( $47.7 \%$ ) happened at home followed by those due to road traffic accidents. Conjunctiva and cornea were the most frequently involved ocular structures. Out of 256 patients, 126 (49.2\%) had conjunctival injury while 73 (28.5 $\%)$ had corneal injury. Corneal foreign bodies were seen in 32 patients (12.5\%). Only two patients $(0.8 \%)$ had open globe injury, whereas others had closed globe injury. Eye lids were injured in 45 patients $(17.6 \%)$ and 51 patients $(19.9 \%)$ had injury in the periorbital area. Orbital bone injuries were detected in 16 patients $(6.3$ $\%)$ and was confirmed using CT scan.

\section{CONCLUSIONS}

Ocular trauma is an important cause of preventable blindness and ocular morbidity. It causes high economic burden to the individual as well as to the society. Most of the injuries were closed globe injuries and affected the younger population.

\section{KEY WORDS}

Ocular \& Orbital Trauma, Closed Globe Injury, Open Globe Injury
Corresponding Author: Dr. Viswakala VS, Assistant Professor, Department of Community Medicine, Government TD Medical College, Alappuzha, Kerala, India.

E-mail: drviswakala@gmail.com

DOI: $10.14260 / j e m d s / 2021 / 261$

How to Cite This Article:

Mini PA, Viswakala VS, Verghese JT. Orbital and ocular trauma at a tertiary care centre - a clinical profile. J Evolution Med Dent Sci 2021;10(17):1224-1227, DOI: $10.14260 /$ jemds/2021/261

Submission 04-12-2020,

Peer Review 25-02-2021,

Acceptance 04-03-2021,

Published 26-04-2021.

Copyright (c) 2021 Mani P.A. et al. This is an open access article distributed under Creative Commons Attribution License [Attribution 4.0 International (CC BY 4.0)] 


\section{BACKGROUND}

Considering the cost of medical care as well as the loss of income if a person sustains an ocular trauma, it is very important to strengthen the preventive measures. Awareness about the potential risk factors and possible preventive approach can help people.

Many studies available on the profile and risk factors of orbital and ocular trauma have been carried out in developed countries with widely available modern management and treatment facilities. There is scarcity of studies on the profile of ocular trauma from developing and underdeveloped countries. Such studies can play an important role not only in defining the target groups for prevention and education on ocular trauma but also in prognosticating ocular injuries at the time of presentation, prevent many unnecessary surgical procedures, and also help ophthalmologists dealing with ocular trauma in making clinical decisions.

Ocular injuries are classified according to the Birmingham Eye Trauma Terminology System (BETTS). Ocular trauma can be either closed globe injuries with intact corneoscleral wall or open globe injuries with a full thickness wound of the corneoscleral wall. Mechanism of injury can be either blunt force or with a sharp object. When blunt force produces a closed globe injury, we call it as contusion and when it produces an open globe injury it is rupture globe. Laceration is an open globe injury due to a sharp object while lamellar laceration is a closed globe injury with a sharp object. Trauma can injure the bones or soft tissues of the orbit. Soft tissue injuries in the periocular area can produce periorbital ecchymosis or black eye. If it is associated with orbital bone fractures it can produce orbital emphysema also, where air from the paranasal sinuses will escape into the orbital soft tissues. Orbital and ocular injuries can produce damage to the optic nerve either by directly injuring the nerve with fractured bone fragments or due to compression by hematoma or by shearing forces.

Present study intends at providing clinical profile of patients presenting with orbital and ocular trauma in a tertiary care centre in Kerala. The study can help in providing information about various modalities of presentation following orbital and ocular trauma and also about the nature and severity of ocular morbidity due to trauma. Getting more information in this topic will certainly be helpful in adopting the necessary preventive measures among those prone to ocular injuries, especially in developing countries where available data is scarce.

Importance of study lies in the fact that knowledge about clinical presentation of ocular injuries and time lag for seeking medical care from an ophthalmologist will definitely help in understanding the need for strengthening preventive measures and treatment facilities in our country. Moreover, not many studies have been conducted on this topic in our area.

\section{METHODS}

After getting approval from the institutional ethics and research committee (IERC) this descriptive study was conducted among patients with orbital and ocular trauma attending the ophthalmology casualty of a teaching hospital, during the study period of 3 months from August 2017 to October 2017. A total number of 256 patients were included in the study. Patients with orbital and ocular trauma who were not having other serious injuries necessitating immediate transportation to intensive care unit were included. Those patients with serious head injuries, abdominal or chest injuries or major long bone fractures were excluded as it was difficult to elicit history from these patients and also proper ophthalmological work up was difficult for these patients at the time of presentation. A semi structured pro forma was filled by the attending ophthalmologist after ensuring the stabilisation and immediate management of the patient. All patients included in the study were evaluated for the mode of clinical presentation. After detailed ophthalmological examination which included vision testing using Snellen's chart, anterior segment examination using slit lamp, 90D and indirect ophthalmoscopy for fundus examination, injuries were categorised, intra ocular pressure was assessed digitally by palpation method at the time of presentation. CT-scan was advised whenever indicated.

Anterior segment examination under slit lamp included looking for eyelid injury, conjunctival tear, subconjunctival haemorrhage, corneal foreign body, corneal abrasion, laceration of cornea or sclera, hyphema, iris injuries, pupillary abnormalities, lens subluxation or dislocation and other lens injuries. Posterior segment examination was carried out for detection of vitreous haemorrhage, pre or subretinal haemorrhage, retinal tear or detachment, choroidal tear, choroidal haemorrhage, vascular occlusions or optic disc oedema. All the terminologies were according to Birmingham Eye Trauma Terminology study which is easy to understand.

\section{Statistical Analysis}

The data was entered in Microsoft Excel and was analysed using appropriate software and statistical tests. Nominal data was presented as numbers and percentages.

\section{RESULTS}

A total of 256 patients were examined, highest number of persons (91) who sustained orbital and ocular injuries were belonging to the age group of 21 - 40 years (35.5\%), followed by 65 patients $(25.4 \%)$ in $41-60$ years age group. 205 patients (80.1\%) were males and only 51 (19.9\%) were females. Most of the patients sustained mono-ocular injury and among them majority (150 patients or $58.59 \%$ ) had injury in the right eye. Only 105 patients (41.01\%) sustained injury in the left eye. Only one person had injury in both eyes.

Majority had closed globe injuries (254 i.e., $99.2 \%$ ). Only two patients $(0.8 \%)$ had open globe injuries. Most common site of injury was conjunctiva. Out of 256 patients $126(49.2$ $\%$ ) had conjunctival injury, most of them were associated with subconjunctival haemorrhage. Corneal injury was noted in 73 patients $(28.5 \%)$ and corneal foreign bodies were removed from 32 patients $(12.5 \%)$. Six patients had lens injury $(2.3 \%)$, one patient $(0.4 \%)$ had scleral injury, three patients (1.2\%) had hyphema (blood in anterior chamber), 
two $(0.8 \%)$ patients had vitreous haemorrhage and two patients $(0.8 \%)$ developed traumatic optic nerve injury.

Considering the ocular adnexa and orbital area, 14 patients (5.5\%) had eyebrow injury, 15 patients (5.9\%) had periorbital ecchymosis, 45 (17.6\%) patients had eyelid injury and 16 patients (6.3\%) had orbital bone injury. Regarding the time lag between the injury and time of examination in casualty, 242 patients (94.5\%) got medical attention within the initial 6 hours of injury. 11 patients (4.3\%) reached hospital within 6 - 12 hours of injury, two patients $(0.8 \%)$ reached within 12 - 24 hours and only one person $(0.4 \%)$ got delayed medical attention (after 24 hours).

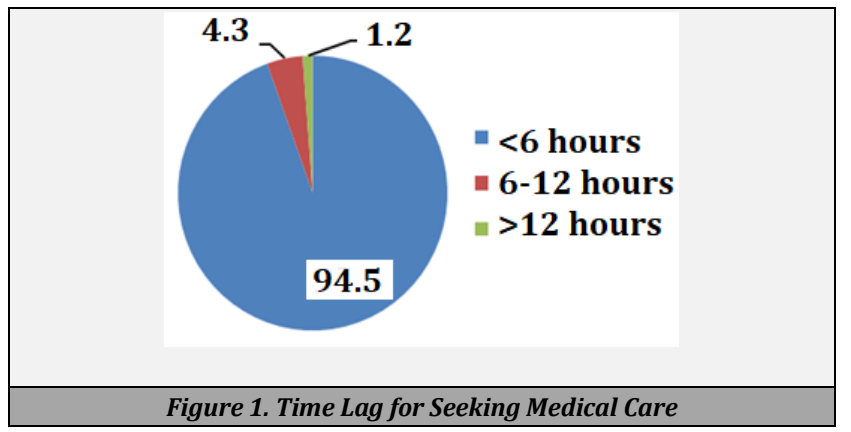

Out of the 256 patients 199 (77.7\%) took outpatient treatment and 47 patients (18.4\%) were admitted in the ophthalmology ward. Four patients $(1.6 \%)$ were referred to higher centre and six patients (2.3\%) were discharged at request against medical advice.

Only 55 patients $(21.5 \%)$ required surgical treatment while 201 patients $(78.5 \%)$ needed medical treatment. About the presenting visual acuity, $205(80.1 \%)$ patients had visual acuity better than 6 / 60 and only 51 patients (19.9\%) had visual acuity less than 6 / 60. Regarding the intraocular pressure, 249 (97.3\%) had normal intraocular pressure and 7 patients $(2.7 \%)$ had abnormal intraocular pressure at the time of presentation. Fundus examination could be done only in 254 patients with closed globe injury. 234 patients (91.4 $\%)$ showed normal fundus, 20 patients $(7.8 \%)$ showed abnormal fundus and in two patients with open globe injury fundus was not visible.

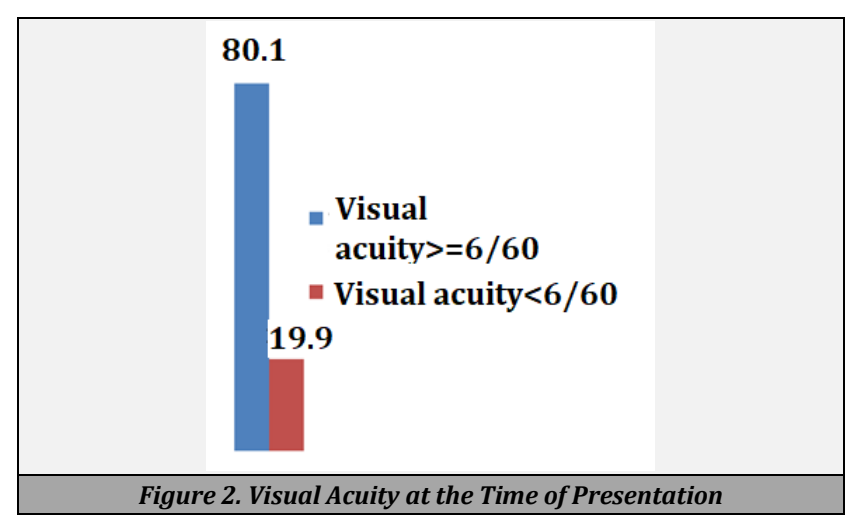

\section{DISCUSSION}

Ocular injuries are considered as a common cause of visual morbidity especially affecting the productive age group of the population. Blindness has regularly been one of the most feared of all disabilities and any threat to vision is emotionally disturbing and will upset physical, mental, social and economic status of the affected. A number of studies were conducted in various parts of the world to determine the epidemiological pattern as well as clinical profile of patients suffering from ocular and orbital trauma. These injuries can occur in any setting such as home, school, workplace or in roads following road traffic accidents. By studying the clinical profile, we can assess the gravity of ocular injury as well as the ocular structures frequently affected. In the present study, out of the total 256 patients $156(60.9 \%)$ were in the age group of 21 - 60 years and was the productive population of the community. Their visual morbidity will indirectly affect the financial status of the family and society.

In our study most of the patients had injury to one eye only, right eye being more commonly affected (58.59\%) compared to left eye (41.01\%). Bilateral injuries were very rare in our study $(0.4 \%)$. This finding is similar to that of the study conducted by Sony Sinha ${ }^{1}$ in a tertiary care centre in Jharkhand where right eye preponderance was seen in 68.4 $\%$ and reported $0.4 \%$ cases with bilateral injury. In a study by Shailaja Karve et al. $^{2}$ at a tertiary eye care centre in rural Maharashtra both right and left eye were involved almost equally, and they found $1.75 \%$ of bilateral injuries. Bhawesh Chandra Saha et al. in their study on paediatric penetrating ocular trauma in a tertiary eye centre in Eastern India, among 86 children,47 (54.7\%) had left eye involvement. ${ }^{3}$

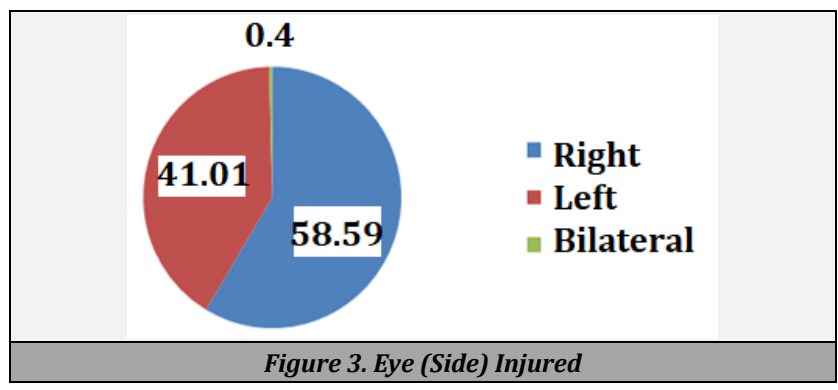

In the present study most common type of injury was closed globe injury (99.2\%) and only two cases of open globe injury (0.8\%) were seen. Most common site involved was conjunctiva (49.2\%), next being cornea (28.5\%). Out of the corneal injuries $32(12.5 \%)$ had corneal foreign bodies. Lens injury was seen in six patients $(2.3 \%)$. Iris injury and Hyphema were seen in 3 patients (1.2\%). Only 2 patients had Optic nerve injury $(0.8 \%) 2$ patients had vitreous haemorrhage $(0.8 \%)$ and 1 patient had retinal injury $(0.4 \%)$. Regarding the orbital and periocular injuries, 16 patients $(6.3$ $\%)$ had orbital bone fractures. 14 patients (5.5\%) had eyebrow injuries and 45 patients (17.6\%) had eyelid injuries. In the study by Shailaja Karve ${ }^{1}$ et al. the most common structure involved was cornea (54\%) followed by conjunctiva (24.25\%) and lens (13.25\%). In the study conducted by M Sunil Kumar ${ }^{4}$ et al. in Karnataka, conjunctiva (80\%) was the most commonly involved ocular structure followed by periorbital lesions (66.6\%). Poy Raiturcar TA, et al. had similar results of the present study with closed globe injuries that were seen among $90 \%$ of patients and nearly half of the patients had conjunctival injuries, i.e., $46.4 \%$ and included subconjunctival haemorrhage, chemosis, and 
conjunctival tears; $23 \%$ of their study subjects had corneal injuries ${ }^{5}$.

The final visual outcome and morbidity depends on nature and extent of injury, the time lag between occurrence of injury and obtaining expert medical help, presence of other comorbidities and type of treatment provided. In the present study most of the patients, 242 out of 256 (94.5\%) reached hospital within 6 hours of injury. This absence of delay may be because of high literacy and health awareness of the people as well as easy availability of medical service in Kerala. Better facilities for transport to the hospital is also available. Only 1 patient $(0.4 \%)$ reached hospital after 24 hours. Shailaja Karve ${ }^{2}$ et al. reported that in their study only $35 \%$ cases reported within 24 hours. Poy Raiturcar TA, et al. in their study conducted in a tertiary care centre in Goa reported that majority, i.e., $77 \%$ presented to the hospital within the first $6 \mathrm{hrs}$ after the injury which is very similar to the present study. ${ }^{5}$ Bhawesh Chandra Saha et al. in their study observed that patients reporting within 24 hours of injury were only $45.5 \%$.

Anterior segment involvement was more common (98\%) in the present study compared to posterior segment which is similar to the various study reports. In study conducted by Shailaja Karve et al. $82.75 \%$ were having anterior segment involvement. ${ }^{2}$ Most serious injuries were seen in open globe injuries. This is consistent with eye injury reported by Shailaja Karve ${ }^{2}$ et al. in their study where $85.04 \%$ were closed globe injuries and $14.96 \%$ were open globe injuries. In the study conducted by Jawade $S$ et al. most of the patients who suffered from blunt ocular trauma had sub conjunctival haemorrhage followed by hyphaema, iritis and traumatic mydriasis. ${ }^{6}$ According to Poy Raiturcar TA, et al. isolated anterior segment injuries were seen in $96.4 \%, 1.4 \%$ had isolated posterior segment injuries, and $2.2 \%$ had combined anterior as well as posterior segment injuries. ${ }^{5}$

In our study 201 (78.5\%) patients were managed medically and $55(21.5 \%)$ were managed surgically. This was comparable with the study conducted by M Sunil Kumar ${ }^{4}$ et al. where $80 \%$ patients were managed medically and $20 \%$ needed surgical treatment. Similarly, in a study conducted in Malawi by Michael Ilsar et al. most of the patients were managed medically ${ }^{7}$. Only 37 out of 102 admitted patients underwent surgical intervention as revealed by a prospective survey of 5671 cases done by Caroline Macewen. ${ }^{8}$

\section{CONCLUSIONS}

This study helps us to understand the clinical presentation of ocular trauma cases. It also reveals the ocular structures involved in the order of frequency as well as the severity of visual impairment among these patients. The commonest type of injury being closed globe injury with anterior segment involvement. This knowledge will help the clinician in more efficient management of ocular trauma cases. A larger study involving primary, secondary, tertiary eye care centres and community-based surveys in these areas will be helpful in filling the gaps of existing knowledge on ocular trauma and its impact in the society.

\section{Limitations of Study}

As the present study did not include patients with severe poly trauma necessitating immediate transportation to ICU, many patients with ocular injuries along with other injuries were excluded from the study.

Data sharing statement provided by the authors is available with the full text of this article at jemds.com.

Financial or other competing interests: None.

Disclosure forms provided by the authors are available with the full text of this article at jemds.com.

\section{REFERENCES}

[1] Sinha S. Pattern of trauma to anterior segment of eyes in a tertiary eye care centre of Jharkhand. J Indian Med Assoc 2008;106(5):289-90.

[2] Karve S, Kolte A, Alfia A, et al. Study of clinical profile of ocular trauma at a tertiary eye care centre. International Journal of Contemporary Medical Research 2017;4(12):77-83.

[3] Saha BC, Kumari R. Clinical profile and management outcome of paediatric penetrating ocular trauma in a tertiary eye centre in eastern India. International Journal of Contemporary Medical Research 2017;4(4):792-4.

[4] Kumar MS, Vishwas D. Clinical profile and visual outcome of ocular injuries presenting to tertiary care hospital located on a state highway. International Journal of Scientific Study 2018;6(5).

[5] Raiturcar TAP, Naik PG, Cacodcar JA. Clinical profile and visual outcome of ocular injuries presenting at a tertiary care hospital in Goa. J Clin Ophthalmol Res 2019;7(2):414.

[6] Jawade S, Dagwar R, Chauhan R. Clinical profile and visual outcome of blunt ocular trauma in a tertiary care centre of central India: a prospective study. International Journal of Medical Science and Clinical Invention 2020;7(11):5063-9.

[7] Ilsar M, Chirambo M, Belkin M. Ocular injuries in Malawi. Br J Ophthalmoml 1982;66(2):145-8.

[8] Macewen CJ. Eye injuries: a prospective survey of 5671 cases. $\mathrm{Br}$ J Ophthalmol 1989;73(11):888-94. 